\title{
The Association between Fibroblast Growth Factor-23 and Vascular Calcification Is Mitigated by Inflammation Markers
}

\author{
Mohamed M. NasrAllah ${ }^{a} \quad$ Amal R. El-Shehaby ${ }^{b} \quad$ Noha A. Osman ${ }^{a}$ \\ Tarek Fayad $^{a}$ Amr Nassef ${ }^{c}$ Mona M. Salem ${ }^{d}$ Usama A.A. Sharaf El Din ${ }^{a}$ \\ Departments of a Nephrology, ${ }^{b}$ Medical Biochemistry, ${ }^{\mathrm{C}}$ Radiology, and ${ }^{\mathrm{d}}$ Endocrinology, \\ Kasr Al-Ainy School of Medicine, Cairo University, Cairo, Egypt
}

\section{Key Words}

Fibroblast growth factor-23 - Vascular calcification - Chronic kidney disease $\cdot$ Inflammation markers · Uremic toxins · Chronic kidney disease

\begin{abstract}
Background: Fibroblast growth factor-23 (FGF-23) has been linked to vascular calcification, ventricular hypertrophy and mortality in chronic kidney disease (CKD), although these links may not be direct and independent. Similar grave outcomes are associated with inflammation and oxidative stress in CKD. Recently, accumulating evidence has linked components of phosphate homeostasis to inflammation and oxidative stress. The interaction between the triad of inflammation, FGF-23 and cardiovascular outcomes is underinvestigated. Methods: We studied 65 patients with stage 5 CKD on hemodialysis. Serum levels of FGF-23, high-sensitivity Creactive protein (hsCRP), endogenous soluble receptor of advanced glycation end products (esRAGE), advanced oxidation protein products (AOPP), parathormone, lipids, calcium and phosphorous were measured. The aortic calcification index (ACI) was determined using noncontrast CT scans of the abdominal aorta. Results: FGF-23 was elevated (mean: 4,681 pg/ml, SD: 3,906$)$ and correlated with hsCRP, esRAGE, AOPP, dialysis vintage and phosphorus in univariate analysis. In multiple regression analysis, hsCRP, AOPP and phosphorus but not esRAGE were all significantly correlated to FGF-23 $\left(R^{2}=0.7, p<0.001\right)$. In univariate analysis, ACI correlated with hsCRP, esRAGE, FGF-23, dialysis vintage, systolic blood pressure (BP) and serum cholesterol. In multiple regression analysis not including inflammation markers, ACI was associated with FGF-23. However, inclusion of inflammation markers in another multiple regression analyses showed that $A C I$ correlated with hsCRP, BP, dialysis vintage and esRAGE but not with FGF-23 $\left(R^{2}=0.65, p<0.001\right)$. Conclusion: FGF-23 is strongly correlated to various markers of inflammation and oxidative stress in hemodialysis patients. The association between FGF-23 and vascular calcification was mitigated when corrected for inflammation markers.
\end{abstract}


NasrAllah et al.: The Association between Fibroblast Growth Factor-23 and Vascula Calcification Is Mitigated by Inflammation Markers

\section{Introduction}

Derangement of mineral metabolism has been incriminated as a harbinger of cardiovascular morbidity and mortality, not only in chronic kidney disease (CKD) but also in the general population [1,2]. With the discovery of fibroblast growth factor-23 (FGF-23) and the plethora of studies published on this phosphaturic agent, some debatable issues have become clearer, while others have become even more confusing [3-5].

Several studies have shown an association between elevated levels of FGF-23 in CKD and left ventricular hypertrophy [6], vascular calcification [7] and mortality [8]. Antagonizing the effects of this hormone through a blockade of its actions has yielded conflicting results regarding its potential clinical benefits. The debate on the role of elevated FGF-23 in CKD as whether it is a physiologically beneficial adjustment, an ominous phenomenon or simply an innocent bystander continues $[5,6,9]$. Although the association between FGF-23 and vascular calcification has repeatedly been demonstrated [7,10-12], it is probably not a direct causeeffect relationship $[13,14]$.

The role of inflammation in the pathogenesis of several complications of CKD is well documented [15]. In particular, several markers have been associated with cardiovascular morbidity and vascular calcification, including endogenous soluble receptor of advanced glycation end products (esRAGE), C-reactive protein (CRP), IL-6 and markers of oxidative stress, with some evidence of a cause-effect relationship [16-18].

Levels of FGF-23 in CKD are elevated and correlate with only a few biological markers related to components of mineral metabolism and renal dysfunction $[7,19]$. Recently, observations from a few experimental and even fewer clinical studies have proposed a possible link between FGF-23 and inflammation, yet the results were not always uniform. However, the link between the triad of FGF-23, inflammation and clinical outcomes remains largely unexplored [20-23].

In this study, we investigate the relationship of FGF-23 with markers of inflammation/ oxidant stress in hemodialysis patients. We also describe the impact of this relationship on the robust link between FGF-23 and vascular calcification that we previously reported in the same group of patients [7].

\section{Methods}

We included 65 patients on a dialysis schedule of $4 \mathrm{~h} /$ session 3 times a week, who were on regular hemodialysis with polysulfone dialyzers and a dialysate calcium concentration of $1.5 \mathrm{~mm} / \mathrm{l}$. The same patients were also included and described in more detail in 2 previous studies investigating the association between FGF-23 [7] and vascular calcification as well as esRAGE [18] and vascular calcification. Patients who had diabetes, a history of parathyroidectomy or were on oral anticoagulation were excluded. Blood samples were drawn in 3 separate tubes for further testing before dialysis and stored at $-70^{\circ} \mathrm{C}$. Whenever a serum sample was thawed for testing, the remaining serum was discarded afterwards. The medical history, prescriptions over the 3 preceding months and mean blood pressure (BP) recordings were registered.

Blood was tested using routine assays for calcium, phosphorus, lipid profile and albumin. The serum levels of intact parathyroid hormone were measured with an enzyme-amplified sensitivity immunoassay (Roche Diagnostics, Indianapolis, Ind., USA). Intact FGF-23 was measured with a two-site (NH2-terminal/C-terminal) ELISA (Immutopics, San Clemente, Calif., USA), esRAGE with an ELISA-based kit (Daiichi Fine Chemical Co. Ltd., Takaoka, Japan, distributed by B-Bridge International Inc.), high-sensitivity CRP (hsCRP) with ELISA (Oxis 
Table 1. Characteristics of the study population
NasrAllah et al.: The Association between Fibroblast Growth Factor-23 and Vascular Calcification Is Mitigated by Inflammation Markers

\begin{tabular}{lc}
\hline Age, years & $50 \pm 11.5(50 ; 19-73)$ \\
Males & $28(43)$ \\
Smokers & $15(23)$ \\
Dialysis vintage, months & $31.7 \pm 39.8(18 ; 0.1-204)$ \\
Systolic BP, mm Hg & $150 \pm 20.2(150 ; 90-190)$ \\
Diastolic BP, mm Hg & $91 \pm 11.3(90 ; 60-110)$ \\
Cholesterol, mg/dl & $154.9 \pm 52(146 ; 36-323)$ \\
Triglycerides, mg/dl & $127 \pm 63.9(102 ; 50-349)$ \\
Alphacalcidol* & $19(29.2)$ \\
Sevelamer, cinacalcet* & 0 \\
Erythropoietin* & $35(53.8)$ \\
CaCO ${ }^{*}$ & $46(70.7)$ \\
Calcium, mg/dl & $8.9 \pm 1(8.9 ; 6.2-10.8)$ \\
Phosphate, mg/dl & $6.8 \pm 2.7(6.2 ; 3.1-16.7)$ \\
Hemoglobin, g/dl & $10.1 \pm 1.3(10.6 ; 8.5-12.3)$ \\
Albumin, g/dl & $3.4 \pm 0.6(3.4 ; 1.9-4.6)$ \\
Parathormone, pg/ml & $314.7 \pm 368.2(169.4 ; 13.2-1,900)$ \\
ACI, \% & $14.1 \pm 12(11.7 ; 0-67.5)$ \\
hsCRP, $\mu \mathrm{g} / \mathrm{ml}$ & $6.8 \pm 4.7(5.3 ; 0.45-21)$ \\
FGF-23, pg/ml & $4,681.3 \pm 3,906.1(4,058 ; 229-13,256.7)$ \\
AOPP, $\mu \mathrm{M} / \mathrm{l}$ & $133 \pm 103(109.4 ; 1-448)$ \\
esRAGE, pg/ml & $278 \pm 101.1(278 ; 145-489)$ \\
\end{tabular}

Figures are reported as mean \pm SD (median; range) or $\mathrm{n}(\%)$. * Number of patients using the drug over the 3 preceding months.

International Inc., Beverly Hills, Calif., USA) and advanced oxidation protein products (AOPP) was measured with spectroscopic analysis (Immundiagnostik, Bensheim, Germany).

The aortic calcification index (ACI) was determined semiquantitatively using noncontrast axial CT scans of the abdominal aorta, as previously detailed [7]. Ten consecutive slices of the abdominal aorta were subdivided radially into 12 sectors at 1 -cm intervals. The sectors showing areas of calcification $(\geq 100 \mathrm{HU}$ ) were counted, divided by 120 and then multiplied with 100 to express them as percentages. One of the examiners (M.M.N.) interpreted the results with an intraobserver variability of $3.7 \%$.

After the patients had been informed about the study procedures, they gave informed consent for participation, and approval from the local research ethics committee was obtained.

The statistical package for the social sciences (SPSS; version 7.5) was used for data analysis, and the data were summarized as mean \pm SD and median, range. Comparisons between the groups were made with the Student t test, and the Mann-Whitney U test was used for the analysis of nonsymmetrically distributed data. Spearman's correlation was used for bivariate analysis, and stepwise multiple regression was performed to correct for possible confounders.

\section{Results}

Characteristics of the study participants are shown in table 1.

\section{Fibroblast Growth Factor-23}

The level of FGF-23 was 4,681 $\pm 3,906 \mathrm{pg} / \mathrm{ml}$. As reported previously, this level was more than 40 times higher than that of a matched control group without kidney disease [7]. In univariate analysis, FGF-23 levels correlated with ACI $(\mathrm{R}=0.48, \mathrm{p}<0.0001)$, dialysis vintage 
Table 2. Stepwise multiple regression analysis of factors correlating with FGF-23, $\mathrm{R}^{2}=0.68$ (corrected for phosphorus, hsCRP, AOPP, esRAGE and dialysis vintage)

\begin{tabular}{llcc}
\hline & $\beta$ & $\mathrm{p}$ & $95 \% \mathrm{CI}$ \\
\hline hsCRP & 0.6 & $<0.0001$ & $387-634$ \\
AOPP & 0.4 & $<0.0001$ & $8-20$ \\
Phosphorus & 0.3 & $<0.0001$ & $273-733$ \\
\hline
\end{tabular}

Table 3. Stepwise multiple regression analysis of factors correlating with ACI, $\mathrm{R}^{2}=0.65$ (corrected for hsCRP, systolic BP, esRAGE, AOPP, FGF-23, dialysis vintage and age)

\begin{tabular}{lccc}
\hline & $\beta$ & $\mathrm{p}$ & $95 \%$ CI \\
\hline hsCRP & 0.47 & $<0.0001$ & 0.7 to 1.7 \\
Systolic BP & 0.33 & $<0.0001$ & 0.1 to 0.3 \\
esRAGE & -0.3 & 0.001 & -0.06 to -0.014 \\
Vintage & 0.16 & 0.04 & 0.06 to 0.1 \\
\hline
\end{tabular}

$(\mathrm{R}=0.6, \mathrm{p}<0.0001)$ and phosphorus $(\mathrm{R}=0.5, \mathrm{p}<0.0001)$. In addition, all measured inflammatory markers correlated with FGF-23 (hsCRP: $\mathrm{R}=0.6, \mathrm{p}<0.0001$; esRAGE: $\mathrm{R}=-0.55, \mathrm{p}<$ 0.0001; AOPP: $\mathrm{R}=0.44, \mathrm{p}<0.0001$ ). Stepwise multivariate analysis accounting for the correlating factors vintage, phosphorus, AOPP, esRAGE and hsCRP revealed that markers of inflammation and oxidative stress were more robustly correlated to FGF-23 than phosphorus (table 2).

\section{Aortic Calcification Index}

Calcification of the abdominal aorta was detectable in 64 out of 65 patients and showed no relation to the intake of calcium-based phosphate binders or alphacalcidol. In univariate analysis, ACI was negatively associated with esRAGE $(\mathrm{R}=-0.6, \mathrm{p}<0.0001)$ and positively correlated with hsCRP $(\mathrm{R}=0.67, \mathrm{p}<0.0001), \mathrm{FGF}-23(\mathrm{R}=0.48, \mathrm{p}<0.0001)$, dialysis vintage $(\mathrm{R}=0.44, \mathrm{p}<0.0001)$, systolic $\mathrm{BP}(\mathrm{R}=0.4, \mathrm{p}=0.001)$ and serum cholesterol $(\mathrm{R}=0.27, \mathrm{p}=$ $0.027)$.

Three models of stepwise multiple regression analysis were employed to adjust for the factors correlating with aortic calcification. The first model, also reported in a previous study, was corrected for FGF-23, age, dialysis vintage, BP, parathormone, phosphorus, triglycerides and cholesterol. In this model $\left(\mathrm{R}^{2}=0.48\right)$, the independent parameters associated with ACI were FGF-23 ( $\beta=0.6, \mathrm{p}<0.001)$ and systolic BP [7].

The second model used in the current study, was a regression model that included the factors correlating with aortic calcification as well as inflammatory markers (FGF-23, esRAGE, hsCRP, AOPP, dialysis vintage and systolic BP) and age. After adjustment for inflammatory markers, the correlation of FGF-23 with ACI reduced the standardized regression coefficient to insignificant levels ( $\beta=0.1, p=0.3$; table 3 ). In a third model, we only corrected for hsCRP and FGF-23, and again, FGF-23 showed no significant correlation with ACI after correction for hsCRP $\left(R^{2}=0.5\right.$; hsCRP: $\beta=0.6, p<0.0001$; FGF-23: $\left.\beta=0.15, p=0.2\right)$. 
NasrAllah et al.: The Association between Fibroblast Growth Factor-23 and Vascula Calcification Is Mitigated by Inflammation Markers

\section{Discussion}

We showed that FGF-23 was correlated to markers of inflammation and oxidative stress in end-stage renal disease patients on hemodialysis. Unlike what we demonstrated in a previous study on the same patients, the correlation of FGF-23 with aortic calcification did not seem to be independent, as it was mitigated by the correction for inflammation markers.

FGF-23 was repeatedly shown to be strikingly elevated in patients with CKD, and only few clinical markers were associated with this elevation, namely phosphorus, CKD stage and dialysis vintage $[7,19,24]$. The correlation between FGF-23 and inflammation may be a new explanation for the high levels of FGF-23 in CKD patients. Only few clinical studies have described the relation between FGF-23 and inflammatory markers. To date, Mendoza et al. [20] found the most convincing evidence of a positive association between FGF-23 and IL-6, CRP and TNF- $\alpha$ in predialysis CKD patients because findings from other studies were less consistent [22] or even contradictory [21]. The regression analysis (table 2) conducted in the current study showed that FGF-23 correlated well with different markers of inflammation and oxidative stress. This correlation was even more robust than the well-established correlation with phosphorus that was previously reported by us as well as other researchers [7, $11,19,24]$.

On the other hand, the association between inflammatory markers and vascular calcification is frequently reported $[25,26]$. Particularly advanced glycation end products provide experimental evidence of a possible cause-effect relationship with vascular calcification [16, 25], and esRAGE may have a protective role [18].

The question that has not been approached yet is whether the interaction between inflammation and FGF-23 has an impact on the sinister associations of FGF-23 [20, 26]. Along with others, we had previously shown an independent association between FGF-23 and aortic calcification $[7,11]$. The regression analysis presented here showed that adjusting for inflammatory and oxidative stress markers simply excluded FGF-23 from the independent variables correlating with aortic calcification (table 3). Indeed, the association between FGF-23 and vascular calcification may not be an independent, genuine association as suggested by recent studies in which FGF-23 failed to induce vascular calcification in vitro [13, 14].

The molecular and pathophysiological links between FGF-23 and inflammation still need to be studied in more detail. It is possible that FGF-23 is capable of stimulating and/or accentuating inflammatory cascades. FGF-23-responsive genes include several genes that encode proinflammatory cytokines [27]. FGF-23 activates ERK-1 and phospholipase C- $\gamma$ isozyme pathway signalling independently of Klotho [6]. Interestingly, these pathways are also capable of signalling an increased production of IL-6, IL-8 and TNF- $\alpha[27,28]$. The second possibility is that inflammation increases FGF-23 levels. Several cytokines and inflammatory pathways, including TNF- $\alpha$ and NF- $\kappa$ B, can inhibit bone matrix PHEX and DMP-1, possibly resulting in an increased FGF-23 production from osteocytes [29-31]. A third possibility is that both inflammation and FGF-23 are aroused simultaneously by an extrinsic signal, conceivably phosphorus. Phosphorus has the capacity to increase FGF-23 production as well as stimulate inflammatory cascades and oxidative stress [24, 32, 33]. Other links between inflammation and FGF-23 could be via vitamin D or involvement of signalling pathways in the liver and adipose tissue producing cytokines and acute-phase reactants [20]. To complete the loop, all hypothesized pathways involving FGF-23, phosphorus, inflammation, PHEX, DMP-1, vitamin $D$, etc., are also pathways that influence bone mineralization and can affect vascular calcification directly or indirectly $[29,30]$. It is possible that these multifaceted pathways interlace with each other in complex feedback interactions, affecting bone mineralization, vascular calcification and inflammation in CKD. 
In this study, we reported the association between FGF-23 and 3 inflammation markers, namely (a) AOPP, a marker of oxidative stress, (b) hsCRP, a nonspecific, acute-phase marker of inflammation, and (c) esRAGE, a surrogate marker of oxidative stress and several other inflammatory cascades. These inflammatory markers are also surrogates of pathways previously shown to be associated with vascular calcification $[18,25,26,34]$. The main drawbacks of this study are the small number of patients and the cross-sectional design; however, we tried to overcome these weaknesses by using a sensitive radiological technique for the diagnosis of vascular calcification to increase the accuracy of the collected data. The most pertinent finding of this study was that the strong relationship we previously demonstrated between FGF-23 and vascular calcification in the same patients [7] was weakened when corrected for inflammation markers. This is, to our knowledge, the first report to demonstrate an interaction between inflammation and the ominous associations of FGF-23. The nature of this interaction needs to be elucidated by experimental and longitudinal clinical studies to clarify whether there is a cause-effect relationship or not.

\section{Disclosure Statement}

The authors have no conflicts of interest to declare regarding the contents of this study.

\section{References}

1 Donate-Correa J, Muros-de-Fuentes M, Mora-Fernández C, Navarro-González JF: FGF23/Klotho axis: phosphorus, mineral metabolism and beyond. Cytokine Growth Factor Rev 2012;23:37-46.

2 Gonzalez-Parra E, Tuñón J, Egido J, Ortiz A: Phosphate: a stealthier killer than previously thought? Cardiovasc Pathol 2012;21:372-381.

3 Wolf M: Forging forward with 10 burning questions on FGF23 in kidney disease. J Am Soc Nephrol 2010;21: 1427-1435.

4 Wolf M: Update on fibroblast growth factor 23 in chronic kidney disease. Kidney Int 2012;82:737-747.

5 Ketteler M, Biggar PH, Liangos O: FGF23 antagonism: the thin line between adaptation and maladaptation in chronic kidney disease. Nephrol Dial Transplant 2013;28:821-825.

6 Faul C, Amaral AP, Oskouei B, et al: FGF23 induces left ventricular hypertrophy. J Clin Invest 2011;121:43934408.

7 Nasrallah MM, El-Shehaby AR, Salem MM, Osman NA, El Sheikh E, Sharaf El Din UA: Fibroblast growth factor-23 (FGF-23) is independently correlated to aortic calcification in haemodialysis patients. Nephrol Dial Transplant 2010;25:2679-2685.

8 Gutierrez OM, Mannstadt M, Isakova T, et al: Fibroblast growth factor23 and mortality among patients undergoing hemodialysis. N Engl J Med 2008;359:584-592.

9 Shalhoub V, Shatzen EM, Ward SC, et al: FGF23 neutralization improves chronic kidney disease-associated hyperparathyroidism yet increases mortality. J Clin Invest 2012;122:2543-2553.

10 Masai H, Joki N, Sugi K, Moroi M: A preliminary study of the potential role of FGF-23 in coronary calcification in patients with suspected coronary artery disease. Atherosclerosis 2013;226:228-233.

11 Jean G, Bresson E, Lorriaux C, et al: Increased levels of serum parathyroid hormone and fibroblast growth factor-23 are the main factors associated with the progression of vascular calcification in long-hour hemodialysis patients. Nephron Clin Pract 2012;120:c132-c138.

12 Coen G, De Paolis P, Ballanti P, et al: Peripheral artery calcifications evaluated by histology correlate to those detected by CT: relationships with fetuin-A and FGF-23. J Nephrol 2011;24:313-321.

13 Scialla JJ, Lau WL, Reilly MP, et al: Fibroblast growth factor 23 is not associated with and does not induce arterial calcification. Kidney Int 2013;83:1159-1168.

14 Lindberg K, Olauson H, Amin R, et al: Arterial klotho expression and FGF23 effects on vascular calcification and function. PLoS One 2013;8:e60658.

15 Stenvinkel P, Heimbürger 0, Lindholm B, Kaysen GA, Bergström J: Are there two types of malnutrition in chronic renal failure? Evidence for relationships between malnutrition, inflammation and atherosclerosis (MIA syndrome). Nephrol Dial Transplant 2000;15:953-960.

16 Yamagishi S, Fujimori H, Yonekura H, Tanaka N, Yamamoto H: Advanced glycation endproducts accelerate calcification in microvascular pericytes. Biochem Biophys Res Commun 1999;258:353-357. 
NasrAllah et al.: The Association between Fibroblast Growth Factor-23 and Vascula Calcification Is Mitigated by Inflammation Markers

17 Sage AP, Tintut Y, Demer LL: Regulatory mechanisms in vascular calcification. Nat Rev Cardiol 2010;7:528536.

18 Nasrallah MM, El-Shehaby AR, Osman NA, Salem MM, Nassef A, El Din UA: Endogenous soluble receptor of advanced glycation end-products (esRAGE) is negatively associated with vascular calcification in non-diabetic hemodialysis patients. Int Urol Nephrol 2012;44:1193-1199.

19 Liu S, Quarles LD: How fibroblast growth factor 23 works. J Am Soc Nephrol 2007;18:1637-1647.

20 Mendoza M, Isakova T, Ricardo AC, et al: Fibroblast growth factor 23 and inflammation in CKD. Clin J Am Soc Nephrol 2012;7:1155-1162.

21 Braithwaite V, Prentice AM, Doherty C, Prentice A: FGF23 is correlated with iron status but not with inflammation and decreases after iron supplementation: a supplementation study. Int J Pediatr Endocrinol 2012; 2012:27.

22 Ashikaga E, Honda H, Suzuki H, et al: Impact of fibroblast growth factor 23 on lipids and atherosclerosis in hemodialysis patients. Ther Apher Dial 2010;14:315-322.

23 Manghat P, Fraser WD, Wierzbicki AS, Fogelman I, Goldsmith DJ, Hampson G: Fibroblast growth factor-23 is associated with C-reactive protein, serum phosphate and bone mineral density in chronic kidney disease. Osteoporos Int 2010;21:1853-1861.

24 Seiler S, Heine GH, Fliser D: Clinical relevance of FGF-23 in chronic kidney disease. Kidney Int Suppl 2009; 114:S34-S42.

25 Wei Q, Ren X, Jiang Y, Jin H, Liu N, Li J: Advanced glycation end products accelerate rat vascular calcification through RAGE/oxidative stress. BMC Cardiovasc Disord 2013;13:13.

26 Stern L: Fibroblast growth factor 23, cardiovascular disease, and inflammation. Clin J Am Soc Nephrol 2012; 7:1061-1062.

27 Dai B, David V, Martin A, et al: A comparative transcriptome analysis identifying FGF23 regulated genes in the kidney of a mouse CKD model. PLoS One 2012;7:e44161.

28 Park JS, Lee EJ, Lee JC, Kim WK, Kim HS: Anti-inflammatory effects of short chain fatty acids in IFN-gammastimulated RAW 264.7 murine macrophage cells: involvement of NF-kappaB and ERK signaling pathways. Int Immunopharmacol 2007;7:70-77.

29 Rowe PS: Regulation of bone-renal mineral and energy metabolism: the PHEX, FGF23, DMP1, MEPE ASARM pathway. Crit Rev Eukaryot Gene Expr 2012;22:61-86.

30 Quarles LD: FGF23, PHEX, and MEPE regulation of phosphate homeostasis and skeletal mineralization. Am J Physiol Endocrinol Metab 2003;285:E1-E9.

31 Uno JK, Kolek OI, Hines ER, et al: The role of tumor necrosis factor alpha in down-regulation of osteoblast Phex gene expression in experimental murine colitis. Gastroenterology 2006;131:497-509.

32 Zhao MM, Xu MJ, Cai Y, et al: Mitochondrial reactive oxygen species promote 65 nuclear translocation mediating high-phosphate-induced vascular calcification in vitro and in vivo. Kidney Int 2011;79:1071-1079.

33 Navarro-González JF, Mora-Fernández C, Muros M, Herrera H, García J: Mineral metabolism and inflammation in chronic kidney disease patients: a cross-sectional study. Clin J Am Soc Nephrol 2009;4:1646-1654.

34 Marinelli A, Orlandi L, Stivali G: C-reactive protein levels are associated with arterial media calcification in nondiabetic patients with end-stage renal disease on long-term hemodialysis. Clin Nephrol 2011;76:425-434. 